\title{
Propuesta metodológica para determinar el potencial de humedad de un material granular a partir de la humedad relativa
}

Jaime Ernesto Díaz Ortiz*

* Ph.D. Profesor - Escuela de Ingeniería de Recursos Maturales y del Ambiente - EIDEMAR - Facultad de Ingeniería - Universidad del Valle - Santiago de Cali, Colombia. E-mail:jaidiaz@univalle.edu.co
Fecha de recepción: Abril 28 de 2004 Fecha de aprobación: Junio 10 de 2005

\section{RESUMEN}

El estudio determina una relación entre el potencial higroscópico y el contenido de humedad de diferentes materiales granulares a partir de la humedad de equilibrio.

Se usaron cámaras selladas para obtener la humedad de equilibrio producida por ácido sulfúrico diluido al 3.3 en volumen, lo cual permitió alcanzar una humedad relativa del 98\%. Se utilizaron diferentes materiales granulares para establecer relaciones entre humedad relativa, contenido de agua en el material granular y potencial higroscópico. Los resultados experimentales mostraron que la humedad de 
equilibrio en los materiales usados se alcanzó aproximadamente en el mismo periodo (13 días), permitiendo establecer correlaciones lineales entre el contenido de agua en el suelo y el potencial higroscópico. El coeficiente de regresión lineal para los valores de potencial obtenidos de los distintos sustratos fue de 0.97.

Palabras Clave: Potencial higroscópico, material granular, sustratos.

\section{ABSTRACT}

This methodology obtains the hygroscopic potential, from the balance between the relative humidity of the atmosphere and the moisture content of humidity of the granular material.

Sealed chambers were used to get the moisture balance generated by the action of sulphuric acid with a dilution of 3.3 in volume, which allows reaching a relative humidity of $98 \%$. Different substrates were used to establish relationships among the relative humidity, the content of water in the soil and the hygroscopic potential. The results found show that the humidity balance of the substrates is reached approximately at the same time, and this fact permitted to establish the appropriate lineal correlations between content of water in the ground and hygroscopic potential. The coefficient of lineal regression obtained for the potential values of the different soils and salts was 0.97 .

Key Words: Hygroscopic potential, granular material.

\section{INTRODUCCIÓN}

Un material granular puede ser cualquier sustancia inerte o no, compuesta por partículas de diferente tamaño, forma y composición química. En ese sentido un suelo, un fertilizante o un volumen de granos o partículas de cualquier material son considerados como materiales granulares. Se define un volumen de material granular como una masa porosa, constituida por partículas de diferente tamaño agrupadas de tal forma que permiten la existencia de un espacio intersticial, llamado también ínter granular. (Puzzi, 1986).

La higroscopicidad es una propiedad que poseen los materiales granulares que depende de la porosidad del material y está definida como la capacidad para absorber o ceder la humedad del ambiente que lo rodea. El equilibrio higroscópico ocurre cuando una masa de material granular con un contenido de humedad dado, alcanza el equilibrio con el aire, a una determinada humedad relativa y a una misma temperatura. Bajo estas condiciones se obtiene la humedad relativa de equilibrio (HRE) en el aire y la humedad relativa de equilibrio (HE) en el producto.

La higroscopicidad condiciona el comportamiento de algunos materiales, por ejemplo en un volumen de granos almacenados, la capacidad de preservación de éstos depende de la interacción entre variables físicas, químicas y biológicas, algunas de los cuales dependen principalmente del medio que los rodea. En este sistema el equilibrio se alcanza cuando el aire y el material granular igualan sus presiones de vapor, de tal manera que no hay migración de la humedad en ningún sentido. En los fertilizantes, una higroscopicidad elevada facilita la disolución de los mismos. En los suelos, la capacidad higroscópica está íntimamente relacionada con el crecimiento de las plantas por su capacidad para retener o ceder la humedad. (Kramer, 1982).

Es importante resaltar que la humedad de un suelo se encuentra en una condición de equilibrio con la humedad relativa del aire que lo rodea. (Wild, 1992)

En los granos de las diversas especies de cereales, oleaginosas y otros de naturaleza higroscópica; el contenido de humedad varía de acuerdo a las condiciones de temperatura $y$ humedad relativa del medio ambiente donde se encuentran.

\section{Volumen 7 - No. 1 - Julio de 2005}


En los suelos, la humedad higroscópica se define como el contenido de agua que se obtiene al establecer la diferencia de peso entre un suelo seco al aire, y un suelo secado en estufa a 105 $110^{\circ} \mathrm{C}$, referido al peso seco y expresado en porcentaje (Fairbridge, 1979). También se expresa como la máxima cantidad de agua que pueden absorber las partículas del suelo por medio de las fuerzas de adsorción, al ponerse en contacto con una atmósfera saturada de vapor de agua (humedad relativa del 100\%), a una temperatura determinada. (Hillel, 1998).

La cantidad de humedad higroscópica en un material granular varía con el porcentaje de saturación de humedad de la atmósfera. Cuando un material granular se encuentra en equilibrio con una atmósfera perfectamente seca, la humedad higroscópica desaparece por completo pero aumenta a medida que se incrementa la humedad relativa (Robinson, 1960).

La humedad higroscópica expresada como agua higroscópica se presenta en forma de delgadas capas de agua. Debido a esa disposición un material granular puede ganar humedad (absorción) o perder humedad (desorción), de acuerdo a distintas combinaciones de temperatura y humedad relativa del aire que se mantiene en equilibrio con esa temperatura y humedad relativa; ese contenido de humedad es denominado "humedad de equilibrio."

Existen muchos procedimientos para disminuir la higroscopicidad y preservar diferentes tipos de materiales. Entre esos se menciona la mezcla de sucrocarbonatos de calcio pulverizados para prevenir el deterioro del azúcar crudo y refinado. (Álvarezet al, 1991)

\section{REVISIÓN BIBLIOGRÁFICA}

El concepto de actividad del agua $\left(a_{w}\right)$ está relacionado con su potencial químico y en la práctica es igual a la humedad relativa de
$a_{W}=\frac{H \cdot R \cdot \operatorname{deE}(\%)}{100}$

La actividad del agua es un indicador de la disponibilidad de este elemento para la actividad biológica de los microorganismos que se encuentran en contacto con un material granular. Por tanto el análisis de la estabilidad en materiales granulares no inertes debe considerar la relación entre la actividad del agua y la temperatura.

Un material granular con determinado contenido de humedad, a una temperatura dada, presentará un equilibrio con la humedad relativa del aire del espacio ínter granular. Esto significa que la temperatura del aire y del material entrarán en equilibrio, y que la humedad relativa del aire estará determinada por el contenido de humedad y por la temperatura de equilibrio aire-material. Este estado de equilibrio se puede obtener de manera estática o dinámica.

Los valores de la actividad del agua en distintos materiales granulares varían para contenidos variables de humedad y temperatura. En el caso de granos como el maíz, para una temperatura de $30^{\circ}$ y contenido de humedad de 12,5 , la actividad de agua es igual a 0,621. Para ese mismo contenido de humedad, la actividad del agua de la soja es 0,720. En el primer caso, para el maíz la actividad del agua asegura la estabilidad del producto durante el almacenamiento; en el segundo caso, para la soja, el producto no está en condiciones de almacenarse por un período de tiempo largo. El análisis de la actividad del agua es el factor más importante para establecer los contenidos de humedad recomendados para el almacenamiento seguro de los granos.

Además del oxígeno y del dióxido de carbono, una parte relativamente pequeña de la atmósfera del material granular se compone de vapor de agua en fase gaseosa. La importancia del agua en fase de vapor no reside en la cantidad existente en un tiempo dado, sino en los procesos que envuelven el movimiento del agua en estado de vapor y en la 
energía necesaria para evaporar el agua. La energía cinética del agua en la fase gaseosa cuando se encuentra en estado de equilibrio y a una misma temperatura, es mayor que la energía del agua líquida. El valor de esta energía es superior en una cantidad igual al calor de vaporización o condensación del agua (Baver, 1991).

La adsorción de agua en superficies sólidas es de naturaleza electrostática y se origina cuando se produce condensación capilar debida al descenso de la presión de vapor dentro de los capilares del material granular. En una fracción de suelo formada por partículas de arcilla, el mecanismo que retiene el agua, se debe a la unión de las moléculas polares de agua a las cargas de las fases sólidas. (Savage, 1984)

La interacción de cargas de las partículas sólidas con las moléculas polares del agua, confiere al agua absorbida una estructura rígida en la cual el agua dipolar adquiere una orientación determinada por la localización de la carga en los sólidos. Según Low (1961) la capa de adsorción, tiene una estructura semejante a una película de hielo casi cristalina y puede tener un espesor de 10 a $20 \AA$.

La humedad relativa de un lugar se relaciona con el contenido de humedad de un suelo. El punto de inflexión en la representación gráfica de ambos parámetros indica aproximadamente la higroscopicidad producida por la condensación capilar (Dirksen, 1999).

El Bureau of Soils, recomienda el uso de ácido sulfúrico para alcanzar la humedad de equilibrio de un suelo, colocando éste en una cámara cerrada que se satura con una solución que permita establecer la humedad de equilibrio. Robinson (1960), utilizó una disolución del $2 \%$ del ácido sulfúrico de densidad de $1.3321 \mathrm{~g} \cdot \mathrm{cm}^{-3}$ para obtener una saturación del 99\% Brown (1992), trabajando para distintas humedades de equilibrio encontró que el ácido sulfúrico diluido al
$10 \%$, permitía alcanzar una humedad relativa del $90 \%$.

El potencial de agua de un material granular se determina estableciendo situaciones de equilibrio entre la humedad del material y la humedad relativa. El potencial se determina cuando se alcanza la humedad de equilibrio.

Modelos de hidrología forestal se han desarrollado con base en la cuantificación del potencial higroscópico, determinando que la conductancia del dosel disminuye hasta cero cuando el potencial se encuentra por debajo de 1.6 Mpa. (Running et al , 1989).

\section{MATERIALESYMÉTODOS}

Cada uno de los materiales granulares utilizados, se colocó en un recipiente diferente, que se encontraba herméticamente cerrado. Se utilizó un método estático para alcanzar la humedad de equilibro. La generación de las condiciones de humedad en los recipientes, se consiguió colocando una disolución volumétrica de 3.3\% de ácido sulfúrico, que permitió alcanzar una humedad relativa de $98 \%$. Se empleó un testigo para verificar esta condición. Se realizó un diseño experimental de cuatro tratamientos con cinco repeticiones por tratamiento. Cada tratamiento correspondió a un material granular.

Como materiales granulares se utilizaron fracciones de suelo (arcilloso, orgánico) y sales (carbonato y sulfato cálcico). La fracción de suelo arcilloso se separó empleando un tamiz de 0.002 $\mathrm{mm}$, y se verificó empleando el método de Bouyoucos que determina la distribución del tamaño de partículas (DTP). Se prefirió este método menos preciso que el de la pipeta, debido a que la intención del ensayo no era identificar el mayor número de fracciones del suelo, sino más bien buscar una aproximación a la clase textural del mismo. Para eso se tomó una muestra de $25 \mathrm{~cm}^{3}$ de pasta saturada, se le agregó $5 \mathrm{ml}$ de solución dispersante

\section{Volumen 7 - No. 1 - Julio de 2005}


(Tripolifosfato de sodio $\mathrm{Ma}_{5} \mathrm{P}_{3} \mathrm{O}_{10}$ ) y $60 \mathrm{ml}$ de agua. La disolución se agitó y dejo reposar durante 24 horas. Después se pasó la suspensión a una copa de una batidora completando un volumen de 250 $\mathrm{ml}$ con agua. Se agitó durante 2 minutos y luego se pasó a una probeta de $250 \mathrm{ml}$. Inmediatamente se realizó la primera lectura de densidad, se dejó reposar durante una hora y se realizó la segunda lectura, que correspondió a la fracción de arcilla. La fracción de suelo arcilloso se secó en estufa a $105^{\circ} \mathrm{C}$ hasta alcanzar peso constante. Posteriormente se extendió en una capa homogénea de $0.01 \mathrm{~m}$ de espesor que se expuso a la humedad de la atmósfera durante 48 horas. La fracción orgánica se obtuvo del horizonte $A$ de un perfil de un suelo orgánico. El porcentaje de materia orgánica total de este suelo fue de $22.58 \%$ y se determinó por el método de Walkley-Black (Walkley-Black, 1934). Como sales se utilizaron carbonato cálcico y sulfato cálcico con contenidos de pureza de $99.45 \%$.

La variación de la humedad relativa se realizó utilizando sondas de temperatura y humedad de alta precisión, que se acoplaban a un equipo digital. Las sondas se calibraron con base en la norma DIMISO 9001.

El tamaño de muestras de cada material granular empleado fue $25 \mathrm{~g}$ repartido en 5 unidades de 5 g cada una, pesados con una balanza digital de precisión $\pm 0.01 \mathrm{~g}$. Diariamente se evaluó la variación de humedad de los sustratos, en los distintos desecadores. El contenido de la humedad se determinó gravimétricamente, por diferencia de peso entre la muestra húmeda y seca en estufa a $105^{\circ} \mathrm{C}$ hasta alcanzar peso constante (Reynolds, 1970).

La relación empleada para determinar el contenido de humedad en los distintos materiales granulares fue la siguiente:

$H S=\frac{S_{h}-S_{s}}{S_{s}} .100$

Donde:
$H S$ : es la humedad de la muestra expresada en porcentaje

$S_{h}$ : peso de la muestra húmeda

$S_{s}$ : peso de la muestra seca

La relación para determinar el potencial del agua de un suelo, cuando se conoce la humedad relativa del aire para un sistema en equilibrio entre las diferentes fases de movimiento de vapor de agua se determina por la siguiente expresión.

$\Psi=-10.62 T \log \left(\frac{100}{a_{W}}\right)$

Siendo:

$\Psi$ : potencial del agua expresada en bar

$T$ : temperatura absoluta, expresada en ${ }^{\circ} \mathrm{K}$

$a_{w}$ : humedad relativa del aire en equilibrio con el agua, adimensional.

Esta relación se utilizó para calcular los resultados de potencial presentados en la Tabla 2 .

\section{RESULTADOS YDISCUSIÓN}

La Gráfica 1 muestra la variación de la humedad en los distintos materiales granulares, hasta alcanzar la humedad de equilibro. El análisis de la figura indica que la humedad de equilibrio para todos los tratamientos se alcanzó aproximadamente en el día 13.

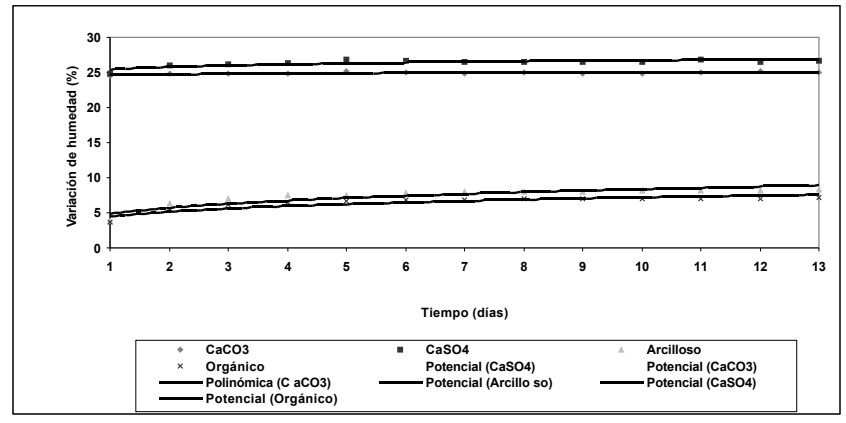

Gráfica 1. Variación del porcentaje de humedad para los distintos materiales en función del tiempo

Los coeficientes de regresión obtenidos para la variación de humedad de los distintos materiales estudiados fueron: 


\begin{tabular}{|c|c|c|c|c|}
\hline Sustrato & $\mathrm{CaCO}_{3}$ & $\mathrm{CaSO}_{4}$ & Arcilloso & Orgánico \\
\hline$R^{2}$ & 0.62 & 0.70 & 0.80 & 0.82 \\
\hline
\end{tabular}

Se observa que los sustratos (sales) con mayor volumen de poros, presentan coeficientes de regresión menores, debido a la dificultad de movimiento de las partículas de vapor de agua en poros de tamaño tan pequeño. Por el contrario los sustratos con menor volumen de poros, pero de mayor tamaño (suelos), presentaron coeficientes de regresión más altos, indicando una variación de incremento constante en estos sustratos.

La diferencia encontrada entre los coeficientes de regresión obtenidos para los distintos materiales granulares empleados en el experimento, puede atribuirse a que la presión de vapor tiene mayor dificultad para llenar con vapor de agua los espacios intersticiales en las sales que tienen poros más pequeños que en los suelos, debido a que el vapor de agua no se comporta como un gas perfecto. La diferencia de contenido de humedad entre ambos sustratos, indica mayor contenido para las sales, debido al mayor volumen de poros.

Los resultados obtenidos muestran que la metodología propuesta para determinar el punto de equilibrio entre la humedad relativa en el interior del recipiente y el contenido de humedad de cada material, presentan una alto grado de correlación. El método se fundamenta en una expresión desarrollada a partir de la ecuación de estado de los gases, que permite calcular el potencial higroscópico de un suelo. Por tanto se considera que el método puede aplicarse a la determinación del potencial higroscópico en otros tipos de materiales porosos y a establecer relaciones adecuadas entre el contenido de humedad y la humedad relativa del ambiente.

Es posible que la metodología propuesta, permita obtener información del potencial higroscópico de los suelos que facilite modelar el movimiento de agua en el suelo, con el fin de predecir variaciones en los niveles de humedad de ecosistemas terrestres o comportamientos de los suelos que valoren las pérdidas de elementos con la escorrentía. Metodologías de este tipo, pueden acoplarse a modelos de circulación general del clima de la Tierra, que permitan predecir fenómenos geoquímicos globales.

En la Tabla 1 se presenta la humedad de los distintos materiales, cuando la disolución de $\mathrm{H}_{2} \mathrm{SO}_{4}$ alcanza la humedad de equilibrio.

Tabla 1. Valores de humedad de equilibrio y contenido de agua de los materiales generados con $\mathrm{H}_{2} \mathrm{SO}_{4}$

\begin{tabular}{|c|c|c|}
\hline Material granular & $\begin{array}{c}\text { Punto de humedad } \\
\text { de equilibrio (g) }\end{array}$ & $\begin{array}{c}\text { Contenido de agua } \\
(\%)\end{array}$ \\
\hline $\mathbf{C a C O}_{3}$ & 25.04 & 2.66 \\
\hline CaSO $_{4}$ & 26.75 & 4.30 \\
\hline Arcilloso & 7.16 & 7.22 \\
\hline Orgánico & 8.29 & 8.23 \\
\hline
\end{tabular}

Con base en los valores obtenidos en el punto de equilibrio de cada uno de los materiales se realizó el cálculo del potencial para los distintos contenidos de agua (Tabla 2).

Tabla 2. Valores de actividad del agua de los materiales y el potencial higroscópico de los materiales.

\begin{tabular}{|c|c|c|}
\hline Material granular & $\begin{array}{c}a_{w} \text { en el Punto } \\
\text { de equilibrio }\end{array}$ & $\begin{array}{c}\text { Potencial } \\
\text { higroscópico (bar) }\end{array}$ \\
\hline $\mathbf{C a C O}_{3}$ & 97.34 & -37.14 \\
\hline $\mathbf{C a S O}_{4}$ & 95.70 & -60.54 \\
\hline Arcilloso & 92.78 & -103.22 \\
\hline Orgánico & 91.77 & -116.30 \\
\hline
\end{tabular}

Los resultados de la Tabla 2, se presentan en la Gráfica 2.

$a_{w}$ en el eje de las abscisas y potencial higroscópico en el eje de las ordenadas.

El coeficiente de regresión lineal obtenido para los materiales utilizados en el ensayo es de 0.97, indicando la bondad de la metodología empleada.

Volumen 7 - No. 1 - Julio de 2005 


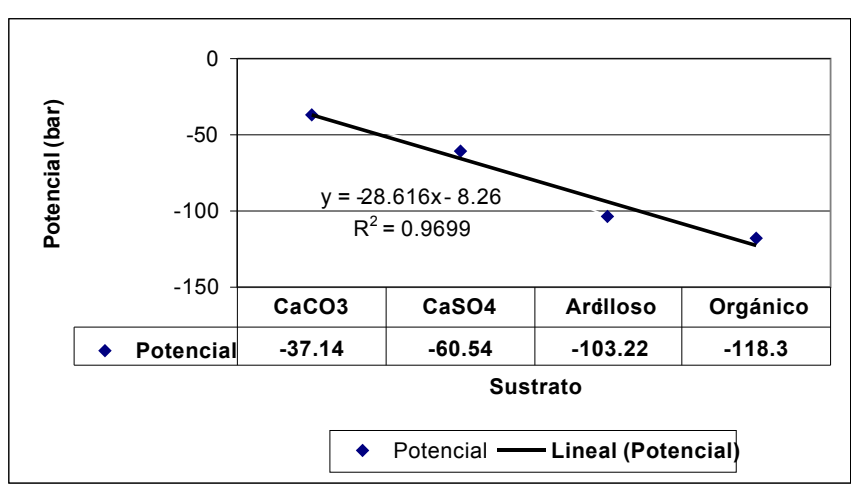

Gráfica 2. Variación del potencial en los distintos materiales

\section{CONCLUSIONES}

$\checkmark$ La metodología propuesta para determinar el potencial higroscópico de un material granular, presenta resultados altamente satisfactorios, al alcanzar un coeficiente de regresión lineal de 0.97 .

$\square \quad$ Los coeficientes de regresión obtenidos para la variación de humedad de los distintos materiales empleados, reflejan adecuadamente el comportamiento de la humedad.

च El método empleado permite establecer relaciones entre la humedad de equilibrio, el contenido de humedad de un material granular y el potencial higroscópico del mismo, para diferentes materiales porosos.

$\square$ Las sales, debido al mayor volumen de poros, presentan los mayores valores de potencial.

$\square \quad$ La metodología propuesta puede ampliarse a diferentes materiales granulares para la determinación del potencial higroscópico.

\section{REFERENCIAS BIBLIOGRÁFICAS}

■ Alvarez Concepción, Mola Emelina; Perera Torres, Pablo ; Burianek, Josef. (1991). Procedimiento para prevenir el deterioro del azúcar crudo y afinado. Invención. Instituto Cubano de Investigaciones Azucareras. Centro Agroindustrial "Pablo Moriega". La Habana, Cuba.

ఐ Baver, L.D.; Gadner, W. H.; Gadner, W. R. (1991). Física de suelos. Editorial Limusa S. A. Mexico. $\square$ Brown, R.W.; Oosterhuis, D. M. (1992).

Measuring plant and soil water potentials with thermocouple psychrometers: some concerns. Agronomy Journal. 84, 78-85.

$\square$ Dirksen, C. (1999). Soll physics measurements. Geoecology paperback. Catena Verlag.

च Fairbridge, Rh. W.; Finkl, CH. W. (1979). The encyclopaedia of soil science. Part 1. Dowden Hutchinson and Ross, Inc.

$\square$ Hillel, D. (1998). Environmental Soil Physics. Academic pres.

$\square$ Kramer, P.J. (1982). Water and plant productivity of yield. pp. 41 - 47. In M. Rechcigl (ed), Handbook of Agricultural Productivity. CRC Press, Boca Raton, Florida.

$\square$ Low, P. F. (1961). Physical chemistry of clay water interactions. Advanced agronomy. 13, 269327.

$\square$ Puzzi, D. (1986). Abastecimento e armazenagem de grãos. Campinas, Sp: Instituto campineiro de encino agrícola.

$\square$ Reynolds, S.G. (1970). The gravimetric method of soil moisture determination. Part I. A study of equipment and methodological problems. Journal of Hydrology, 11: 258-273.

$\square$ Robinson, G. W. (1960). Los suelos, su origen constitución y clasificación Introducción a la Edafología. Traducido por Amoros, J. L. Ediciones omega. Barcelona.

$\square$ Running, S.W., R.R. Memani, D.L. Peterson, L.E. Band, D.F. Potts, L.L. Pierce, and M.A. Spanner. (1989). Mapping regional forest evapotranspiration and photosynthesis by coupling satellite data with ecosystem simulation. Ecology 70: 1090-1101.

$\square$ Savage, M.J.; Cass, A. (1984). Measurement of water potential using in situ thermocouple hygrometers. Advances in Agronomy. 32.

$\square$ Walkley, A..; Black, I. A. (1934). An examination of the Dejtjareff method for determining soil organics matter and a proposed modification of the cromic acid tritation method. Soil Science. 37: 29-38.

$\square$ Wild, A. (1992). Condiciones del suelos y desarrollo de las plantas según Rusell, Ediciones Mundi-Prensa. 